\title{
Influence de la substitution graduelle de tourteau de soja par la féverole sur la croissance et la conformation de la carcasse chez les poulets de chair
}

\author{
OKANDZA Yves 1,2, MOPOUNDZA Paul 1 ., DIMI NGATSE Silvère ${ }^{1}$., HALBOUCHE Miloud² et AKOUANGO \\ Parisse 1 \\ 1. Laboratoire de Zootechnie et Biodiversité, École Nationale Supérieure d'Agronomie et de Foresterie, Université Marien \\ Ngouabi. BP. 69. Brazzaville-Congo. \\ 2. Institut National de Formation Supérieure Agronomique de Mostaganem. Algérie. \\ Auteurs Correspondant s : Professeur Parisse AKOUANGO ; Email : parakouango@yahoo.fr \\ Okandza Yves ; Email : yvesokandza@yahoo.fr
}

Original submitted in on $17^{\text {th }}$ October 2016. Published online at www.m.elewa.orgon $28^{\text {th }}$ February 2017 http://dx.doi.org/10.4314/jab.v110i1.2

\begin{abstract}
RESUME
Objectif : Le présent travail a été entrepris pour déterminer l'influence de la substitution graduelle de tourteau de soja par la féverole sur la croissance et la conformation de la carcasse chez les poulets de chair.

Méthodologie et résultats :Notre essai a été porté sur 50 poussins de souche ISA repartis en 05 lots homogènes et soumis à 05 régimes alimentaires différents par leurs taux d'incorporation de 0, 3, 6, 9 et $12 \%$ de féverole.

Les résultats obtenus ont révélé que les sujets nourris aux régimes $0,3,6$ et $9 \%$ de féverole donnent des performances de croissance et de conformation de carcasse satisfaisantes par rapport aux sujets nourris au régime de $12 \%$ de féverole. D'où l'incorporation de la féverole devrait être plafonnée à des taux inférieurs à 12 $\%$ afin de réduire les charges en tourteau de soja dans les rations alimentaires de poulet de chair.

Conclusion et application des résultats : Cet essai est un objet qui permet d'améliorer les performances de croissance et de conformation de carcasse chez les poulets de chair. Elle montre que l'incorporation de la féverole à des taux inférieurs à $12 \%$ réduit les charges en tourteau de soja dans les rations alimentaires de poulet de chair.
\end{abstract}

Mots clés : Substitution, soja, féverole, performances zootechniques, poulet de chair.

\section{ABSTRACT}

Objective: This work was undertaken to determine the influence of the gradual substitution of soybean meal on carcass growth and conformation in broiler chickens.

Methods and results: Our test was carried out on 50 chicks of ISA strain distributed in 05 homogeneous batches and subjected to 05 different diets by their incorporation rates of $0,3,6,9$ and $12 \%$ of faba beans. The results obtained showed that subjects fed on diets $0,3,6$ and $9 \%$ of faba bean gave satisfactory carcass growth and conformation performances compared to subjects fed on a $12 \%$ faba bean diet. Hence the incorporation of fababeans should be capped at rates below $12 \%$ in order to reduce soybean meal loads in broiler feed rations. 
Conclusion and application of the results: This test is an object, which improves the performance of carcass growth and conformation in broiler chickens. It shows that incorporation of faba beans at rates below $12 \%$ reduces soybean meal loads in broiler feed rations.

Keywords: Substitution, soybeans, faba beans, zootechnical performances, broiler chickens.

\section{INTRODUCTION}

L'alimentation des volailles, plus précisément celle des poulets de chair en Afrique ne cessera d'être l'objet d'étude tant que les besoins humains ne cesseront de croître. La recherche de solutions meilleures permettant d'améliorer la productivité des oiseaux de la basse-cour, une des pistes vers la lutte contre la faim, notamment par des rations alimentaires moins contraignantes est une évidence (Akouango, 2014). Les graines de féverole sont relativement riches en énergie avec une valeur voisine de celle des céréales (Cartoux, 2010). Elles sont également riches en protéines avec une teneur inférieur de près de $50 \%$ à celles du tourteau de soja (Catroux, 2010), donc elle peut substituer le tourteau de soja mais partiellement à cause de la présence de plusieurs facteurs antinutritionnels. Ces protéines sont riches en lysine, mais assez pauvres en acides aminés soufrés et tryptophanes. Vadivel et Pugalenthi, (2010) ont utilisé plusieurs méthodes afin de déterminer la composition de la féverole. A l'aide de la méthode micro Kjeldahl et la méthode soxhelt d'extraction, ils ont déterminé la teneur en protéines brutes et en lipide qui sont respectivement $265 \mathrm{~g}$ et $65 \mathrm{~g} / \mathrm{kg}$ de MS. Ces valeurs sont relativement élevées par rapport à d'autres légumineuses ce qui contribue à utiliser la féverole comme une source protéique alternative pour réduire l'indépendance à la protéine du tourteau de soja. Ils ont rapporté aussi que les acides aminés essentiels sont suffisants à l'exception de la méthionine, tyrosine et cystine et la composition de la féverole en acides aminés est très comparable à celui du soja après des analyses à l'aide de l'analyseur automatique des acides aminés. La févérole représente une bonne source protéique et énergétique, mais réduit les performances lorsqu'elle est utilisée à des niveaux élevés (Benabdeljejil et Boubakraoui, 1985). Le tourteau de soja est riche en matière azotée totale et surtout en lysine. II renferme une grande quantité de protéines, de glucides, de lipides, de vitamines $A$ et $B$, de phosphore, de potassium, de calcium, de magnésium, de zinc et de fer (Metayer et al., 2003 ; Zitari, 2008). Le tourteau de soja est la principale source de protéines pour les volailles, mais très cher pour les pays qui importent. Le pourcentage de tourteau de soja inclus dans les rations ne dépasse pas normalement 20\% (Fernandez et Ruiz Matas, 2003). Un excès de tourteau de soja dans la ration peut provoquer des excréments humides. Le principal problème du soja réside dans la présence de facteurs à activité antitrypsique. Ces facteurs antitrypsiques sont localisés pour la plus part, avec les protéines du soja, c'est-à-dire dans les cotylédons. L'inhibiteur de la trypsine est le facteur anti-nutritionnel posant le plus de problèmes. II perturbe la digestion des protéines et provoque l'augmentation de la taille du pancréas des volailles de 50 à 100\%. Comme la plupart des composés antitrypsiques, ceux du soja sont thermostables (Larbier et Leclercq, 1992 ; Drogoul et al., 2004). La majorité des pays Africains (l'Algérie, le Maroc et la Tunisie), les élevages de poulet de chair, nécessitent le tourteau de soja qui est un incontestable pourvoyeur de protéines (Larbier et Leclercq, 1992 ; Dragoul et al., 2004 ; Zitari, 2008). Malheureusement son importation coûte très chère pour l'économie de ces pays et de l'Afrique Sub-Saharienne. Nombreux travaux ont montré que la féverole (Vicia faba $L$ ) peut être utilisée comme source de protéines dans les rations alimentaires chez les poulets de chair (Franck, 1980 ; Lazaro et al., 2003 ; Zitari, 2008). D'autres sources d'information scientifiques ont démontré que l'apport en protéines par la féverole à des taux inférieurs à $9 \%$ améliore la production des œufs chez les pondeuses (Bougon, 1974; Guillaume, 1974). Par contre il y a d'autres travaux qui indiquent que certaines baisses de performances sont dues d'une part à l'augmentation du taux de d'incorporation de la féverole et d'autre part à la présence des facteurs anti-nutritionnels contenus dans la féverole (Larbier et Leclercq, 2003). II est nécessaire de veiller aux taux d'incorporation de la 
féverole dans les rations des poulets de chair. Utilisant ainsi un régime maiis - soja, nous avons voulu savoir dans quelle mesure, la féverole peut être incorporée dans des régimes d'aliments de poulet de chair et pouvant partiellement remplacer le tourteau de soja. Les sujets (poussins) ont été fournis par l'ORAVIO de Ain - Nouissy (Office National d'Aviculture Ouest) et des éléments composant l'aliment formulé ont été fournis par I'Office National d' Aliment de Bétail (Algérie). Ce

\section{MATÉRIEL ET MÉTHODES}

Animaux : Cinquante (50) poussins d'un jour, non sexés de souche ISA sont repartis en 5 lots homogènes de 10 sujets chacun.

Méthodes : Les lots homogènes sont constitués par le prélèvement au hasard de 3 sujets de chacune des 4 classes de poids vif : $33 g-36 g ; 37 g-40 g ; 41 g-44 g$; $45 \mathrm{~g}-54 \mathrm{~g}$. Huit (08) sujets de chaque lot sont placés dès travail a été réalisé à l'atelier pédagogique d'élevage et de phytotechnie de l'INFSA de Mostaganem en Algérie. Les analyses ont été faites aux laboratoires des départements Zootechnie, Sciences du sol, Technologie Agroalimentaire et Phytotechnie de I'INFSA (Algérie) et au Laboratoire de Zootechnie et Biodiversité de l'École Nationale Supérieure d'Agronomie et de Foresterie - Université Marien Ngouabi(Congo).

le 1er jour dans 2 cages (à raison de 04 sujets par cage) placés au sol et subissent le même régime alimentaire affecté aux autres lots. Ces sujets servent à remplacer les animaux en batterie mortes durant le cycle d'élevage. Le tableau 1 indique la composition chimique de la féverole et du tourteau de Soja.

Tableau 1 : Composition chimique de la féverole et du tourteau de Soja utilisés (BOUGON, 1974)

\begin{tabular}{|l|c|c|}
\hline Caractéristiques & $\begin{array}{c}\text { Féverole } \\
(\boldsymbol{\%})\end{array}$ & $\begin{array}{c}\text { Tourteau de Soja } \\
(\%)\end{array}$ \\
\hline Matière sèche & 87,11 & 88,72 \\
\hline Matière organique & 95,95 & 93,07 \\
\hline Matière grasse & 1,50 & 1,06 \\
\hline Protéine brute & 26 & 45 \\
\hline Cellulose brute & 7,5 & 7,00 \\
\hline
\end{tabular}

Le tableau 2 indique les taux d'incorporation de la féverole au détriment de tourteau de Soja.

Tableau 2 : Taux d'incorporation de la féverole au détriment de tourteau de Soja dans différents régimes alimentaires.

\begin{tabular}{|l|c|c|c|c|c|}
\hline \multirow{2}{*}{ Ingrédients } & \multicolumn{5}{|c|}{ Régimes alimentaires } \\
\cline { 2 - 6 } & $\mathbf{0 F}$ & $\mathbf{3 F}$ & $\mathbf{6 F}$ & $\mathbf{9 F}$ & $\mathbf{1 2 F}$ \\
\hline Maïs & 62,49 & 62,49 & 62,49 & 62,49 & 62,49 \\
\hline Tourteau de Soja & 26,04 & 22,91 & 19,79 & 16,66 & 13,54 \\
\hline Féverole & 0,00 & 3,13 & 6,25 & 9,38 & 12,50 \\
\hline Son fin & 6,25 & 6,25 & 6,25 & 6,25 & 6,25 \\
\hline CMV Chair & 1,04 & 1,04 & 1,04 & 1,04 & 1,04 \\
\hline Antistress & 1,04 & 1,04 & 1,04 & 1,04 & 1,04 \\
\hline Phosphate bicalcique & 2,08 & 2,08 & 2,08 & 2,08 & 2,08 \\
\hline Calcaire & 1,04 & 1,04 & 1,04 & 1,04 & 1,04 \\
\hline Méthionine & 0,02 & 0,02 & 0,02 & 0,02 & 0,02 \\
\hline Total & $100 \%$ & $100 \%$ & $100 \%$ & $100 \%$ & $100 \%$ \\
\hline
\end{tabular}

Légende : $0 \mathrm{~F}$ : taux de la féverole à $0 \% ; 3 \mathrm{~F}$ : taux de la féverole à $3 \% ; 6 \mathrm{~F}$ : taux de la féverole à $6 \%$ $9 \mathrm{~F}$ : taux de la féverole à $9 \% ; 12 \mathrm{~F}$ : taux de la féverole à $12 \%$.

Les cinq régimes ont une composition identique mais différents par le taux de substitution du tourteau de Soja par la féverole. Les taux d'incorporation de substitution de tourteau de Soja sont dans l'ordre décroissant. Les animaux sont pesés le jour de leur arrivée et puis hebdomadairement. L'abattage a eu lieu le 56 ème jour de 
l'élevage. Les paramètres de mensurations et de poids sont mesurés. La longueur de la carcasse est mesurée entre la basse du cou et l'extrémité du bourrelet terminal de la queue. La longueur et le tour de cuisse sont évalués respectivement entre l'articulation supérieure du fémur avec le pelvis et celle du tibia avec le métatarse, ensuite autour du grasset. Le poids vif est obtenu par pesée.

Le Gain de Poids Cumulé (GPC) est évalué par la formule suite :

$$
\mathrm{GPC}(\mathrm{g})=\mathrm{Px}-\mathrm{Po}
$$

GPC : Gain de poids cumulé

$P x$ : Poids vif de poulet mesuré par semaine

Po: Poids vif de poulet mesuré au premier jour d'expérimentation.

$$
V C(g / j)=\frac{G P H(g)}{\text { Nombre de jour de la semaine }}
$$

La vitesse de croissance hebdomadaire est obtenue en mettant en rapport le gain de poids hebdomadaire et le nombre de jour de la semaine.

VC : Vitesse de croissance

$\mathrm{GPH}$ : Gain de poids hebdomadaire

\section{RESULTATS}

Gain de poids cumulé, vitesse de croissance et indice de consommation: Le gain de poids cumulé croît de façon continue pour l'ensemble des lots de la 1ère à la 8 ème. Semaine (tableau 3). On remarque toutefois une
L'indice de consommation (IC) est déterminé par la formule suivante :

$$
I C(g)=\frac{\text { Quantité d'aliment consommé }(g)}{\text { Gatn de potds }(g)}
$$

Le poids de carcasse pleine est obtenu par pesée après l'abattage de l'animal, plumé, étêté et débarrassé de ses pattes. Le poids de carcasse évidée est obtenu par pesée après avoir été débarrassé de son tractus digestif, de son gras abdominal, cœur et foie. Le poids de cœur et du foie sont obtenus par pesée après leurs extraits. Le poids du tractus digestif avec jabot est obtenu par pesée. Le poids de la graisse abdominale est obtenu après pesée des graisses qui enveloppent le gésier et celles entourant les intestins.

Traitement statistique : Après 56 jours d'élevage, les paramètres tels que : gain de poids cumulé, vitesse de croissance, indice de consommation, les paramètres pondéraux de carcasse et paramètres de conformation de la carcasse ont fait l'objet d'un traitement statistique suivant l'analyse de variance. Le test de Newman et Keuls ont permis de grouper et de hiérarchiser les moyennes relatives aux paramètres mesurés.

baisse de gain de poids accumulé du lot des sujets recevant le régime $12 \mathrm{~F}$. Cette baisse est plus marquée par rapport aux autres lots à la 4 ème semaine jusqu'à la 8ème semaine.

Tableau 3 : Influence de la substitution du tourteau de Soja par la féverole sur le gain de poids cumulé

\begin{tabular}{|c|c|c|c|c|c|c|c|}
\hline \multirow{2}{*}{ Semaine } & \multicolumn{7}{|c|}{ Régimes } \\
\cline { 2 - 8 } & OF & $3 \mathrm{~F}$ & $6 \mathrm{~F}$ & $9 \mathrm{~F}$ & $12 \mathrm{~F}$ & $\begin{array}{c}\text { Écart type } \\
\text { résiduel }\end{array}$ & $\begin{array}{c}\text { Signification } \\
\mathrm{F}\end{array}$ \\
\hline & $88,3^{\mathrm{ab}}$ & $95^{\mathrm{a}}$ & $84,5^{\mathrm{ab}}$ & $74,1^{\mathrm{b}}$ & $75,1^{\mathrm{b}}$ & 11,5 & ${ }^{* *}$ \\
\hline 1 & $244,2^{\mathrm{ab}}$ & $265,9^{\mathrm{a}}$ & $264,4^{\mathrm{a}}$ & $236,8^{\mathrm{ab}}$ & $218^{\mathrm{b}}$ & 31,68 & ${ }^{*}$ \\
\hline 2 & $483,5^{\mathrm{a}}$ & $486,66^{\mathrm{a}}$ & $494,8^{\mathrm{a}}$ & $462,1^{\mathrm{ab}}$ & $414,5^{\mathrm{b}}$ & 48,55 & ${ }^{*}$ \\
\hline 3 & $851,9^{\mathrm{a}}$ & $826,2^{\mathrm{ab}}$ & $830,9^{\mathrm{ab}}$ & $793,1^{\mathrm{ab}}$ & $776,2^{\mathrm{b}}$ & 91,85 & ${ }^{*}$ \\
\hline 5 & $1192,7^{\mathrm{a}}$ & $1162,3^{\mathrm{a}}$ & $1143,7^{\mathrm{a}}$ & $1096,6^{\mathrm{a}}$ & $953,5^{\mathrm{b}}$ & 129,52 & ${ }^{*}$ \\
\hline 6 & $1467,8^{\mathrm{a}}$ & $1477,3^{\mathrm{a}}$ & $1430,5^{\mathrm{a}}$ & $1442,6^{\mathrm{a}}$ & $1207,7^{\mathrm{b}}$ & 166,90 & ${ }^{*}$ \\
\hline 7 & $1758,8^{\mathrm{a}}$ & $1732,2^{\mathrm{a}}$ & $1684,3^{\mathrm{a}}$ & $1717,6^{\mathrm{a}}$ & $1513,8^{\mathrm{b}}$ & 146,45 & ${ }^{*}$ \\
\hline 8 & 1985,6 & 1962,8 & 1851,8 & 1917,2 & 1737,6 & 220,42 & NS \\
\hline
\end{tabular}

NS : non significatif

Les valeurs indicées de lettres distinctes sont significativement différentes $(P<0,05)$ au test de Newman et Keuls. 


\section{Okandza et al., J. Appl. Biosci. 2017 Influence de la substitution graduelle de tourteau de soja par la}

féverole sur la croissance et la conformation de la carcasse chez les poulets de chair.

La vitesse de croissance : La vitesse de croissance croît de façon continue pour l'ensemble des lots de la 1ère à la 4 ème semaine. À la suite elle présente une diminution de la 4 ème semaine à la $8^{\text {ème }}$ semaine. Une diminution est marquée dans le lot des sujets recevant le régime $12 \mathrm{~F}$ (tableau 4).

Tableau 4 : Influence de la substitution du tourteau de Soja par la féverole sur la vitesse de croissance hebdomadaire

\begin{tabular}{|c|c|c|c|c|c|c|c|}
\hline \multirow{2}{*}{ Semaine } & \multicolumn{7}{|c|}{ Régimes } \\
\cline { 2 - 8 } & OF & $3 \mathrm{~F}$ & $6 \mathrm{~F}$ & $9 \mathrm{~F}$ & $12 \mathrm{~F}$ & $\begin{array}{c}\text { Écart type } \\
\text { résiduel }\end{array}$ & $\begin{array}{c}\text { Signification } \\
\mathrm{F}\end{array}$ \\
\hline 1 & $12,5^{\mathrm{ab}}$ & $13,5^{\mathrm{a}}$ & $12,00^{\mathrm{ab}}$ & $10,6^{\mathrm{a}}$ & $10,7^{\mathrm{b}}$ & 1,67 & ${ }^{* *}$ \\
\hline 2 & 22,2 & 24,4 & 24,9 & 23,5 & 20,4 & 3,21 & $\mathrm{NS}$ \\
\hline 3 & $34,1^{\mathrm{a}}$ & $31,52^{\mathrm{ab}}$ & $33,6^{\mathrm{a}}$ & $32,1^{\mathrm{ab}}$ & $28,0^{\mathrm{b}}$ & 3,81 & ${ }^{*}$ \\
\hline 4 & $52,6^{\mathrm{a}}$ & $48,5^{\mathrm{ab}}$ & $47,9^{\mathrm{ab}}$ & $49,7^{\mathrm{ab}}$ & $40,5^{\mathrm{b}}$ & 7,77 & ${ }^{*}$ \\
\hline 5 & 48,6 & 47,4 & 44,6 & 43,3 & 36,4 & 9,76 & $\mathrm{NS}$ \\
\hline 6 & 39,2 & 44,9 & 40,9 & 49,4 & 36,3 & 7,30 & $\mathrm{NS}$ \\
\hline 7 & 40,9 & 36,4 & 36,2 & 39,2 & 43,7 & 11,37 & $\mathrm{NS}$ \\
\hline 8 & 31,9 & 28,5 & 23,9 & 32,9 & 32,3 & 13,9 & $\mathrm{NS}$ \\
\hline
\end{tabular}

NS : non significatif

Les valeurs indicées de lettres distinctes sont significativement différentes $(P<0,05)$ au test de Newman et Keuls.

L'indice de consommation : À partir de la 4ème semaine, les sujets recevant les régimes $3 \mathrm{~F}, 6 \mathrm{~F}, 9 \mathrm{~F}$ et $12 \mathrm{~F}$ présentent un indice de consommation élevé par rapport au lot OF (tableau 5). Cet indice de consommation augmente avec le taux d'incorporation de la féverole à l'instar des régimes $9 \mathrm{~F}$ et $12 \mathrm{~F}$. À la 6 ème semaine l'indice de consommation est plus en augmentation dans le régime $\mathrm{OF}$. Cet indice de consommation est en baisse dans les régimes $3 \mathrm{~F}, 6 \mathrm{~F}$ et $9 \mathrm{~F}$.

Tableau 5 : Influence de la substitution du tourteau de Soja par la féverole sur l'indice de consommation

\begin{tabular}{|c|c|c|c|c|c|c|c|}
\hline \multirow[b]{2}{*}{ Semaine } & \multicolumn{5}{|c|}{ Régimes } & \multicolumn{2}{|c|}{ Désignation } \\
\hline & OF & $3 \mathrm{~F}$ & $6 \mathrm{~F}$ & $9 \mathrm{~F}$ & $12 \mathrm{~F}$ & $\begin{array}{c}\text { Écart type } \\
\text { résiduel }\end{array}$ & $\begin{array}{c}\text { Signification } \\
\mathrm{F}\end{array}$ \\
\hline 4 & $1,9^{c}$ & $2^{b}$ & $2^{b}$ & $2,2^{a}$ & $2,2^{2}$ & 0,046 & ** \\
\hline 5 & $2,2^{c}$ & $2,4^{b}$ & $2,1^{\circ}$ & $2,5^{a}$ & $2,6^{\circ}$ & 0,124 & ** \\
\hline 6 & $2,9^{a}$ & $2,4^{b}$ & $2,3^{b}$ & $2,5^{b}$ & $2,8^{b}$ & 0,212 & * \\
\hline 7 & $3^{a}$ & $2,8^{a}$ & $2,8^{a}$ & $2,3^{b}$ & $2,2^{b}$ & 0,178 & * \\
\hline
\end{tabular}

Les valeurs indicées de lettres distinctes sont significativement différentes $(P<0,05)$ au test de Newman et Keuls.

Paramètres pondéraux et conformation de la carcasse : Les résultats sur l'influence de la substitution de tourteau de Soja par la féverole sur les paramètres pondéraux et les mensurations de la carcasse sont transcrits dans le tableau 6. Le taux d'incorporation de la féverole a un effet dépressif sur le poids vif. Cet effet dépressif est plus marqué dans le régime $12 \mathrm{~F}$ de féverole. Les régimes $3 \mathrm{~F}$ ont, un poids à celui de $0 \mathrm{~F}$. Les sujets des régimes $6 \mathrm{~F}, 9 \mathrm{~F}, 12 \mathrm{~F}$ présentent des poids qui s'abaissent en fonction du taux de la féverole. Le poids de carcasse pleine du régime 3 Fest semblable à celui du régime OF. Le poids de carcasse pleine baisse dans le régime $12 \mathrm{~F}$. Le poids de carcasse évidée du lot à régime
3 Fse rapproche à celui du lot témoin, par contre celui des régimes $6 \mathrm{~F}$ et $9 \mathrm{~F}$ sont semblables. La féverole a un effet négatif sur le poids de carcasse évidée. Cet effet négatif est plus accentué dans le régime $12 \mathrm{~F}$ où le poids est léger. Une diminution du poids du foie est remarquable à partir des lots recevant des régimes $3 \mathrm{~F}, 6 \mathrm{~F}, 9 \mathrm{~F}$ et $12 \mathrm{~F}$ par rapport au régime $\mathrm{OF}$. Le poids du gras abdominal est supérieur au lot témoin et diminue chez les autres lots. La longueur de la carcasse des régimes $3 \mathrm{~F}, 6 \mathrm{~F}, 9 \mathrm{~F}$ et $12 F$ sont proches à celle du lot témoin. La longueur de la carcasse accuse une diminution à partir du lot recevant le régime $6 \mathrm{~F}$ jusqu'à $12 \mathrm{~F}$. Quant au tour de la cuisse, le lot $3 \mathrm{Fa}$ enregistré une moyenne supérieure aux autres lots. 


\section{Okandza et al., J. Appl. Biosci. 2017 Influence de la substitution graduelle de tourteau de soja par la}

féverole sur la croissance et la conformation de la carcasse chez les poulets de chair.

Tableau 6: Influence de la substitution du tourteau de Soja par la féverole sur les paramètres pondéraux et de conformation de carcasse

\begin{tabular}{|c|c|c|c|c|c|c|c|}
\hline \multirow{2}{*}{ Paramètres } & \multicolumn{7}{|c|}{ Régimes } \\
\cline { 2 - 8 } & OF & $3 \mathrm{~F}$ & $6 \mathrm{~F}$ & $9 \mathrm{~F}$ & $12 \mathrm{~F}$ & $\begin{array}{c}\text { Écart type } \\
\text { résiduel }\end{array}$ & $\begin{array}{c}\text { Signification } \\
\mathrm{F}\end{array}$ \\
\hline $\begin{array}{c}\text { P.V } \\
\text { Avant } \\
\text { abattage }\end{array}$ & 2031,25 & 2009,37 & 1895 & 1962,5 & 1785 & 220,9791 & $\mathrm{NS}$ \\
\hline PCP & 1719,18 & 1710,62 & 1564 & 1628,67 & 1432,5 & 213,3194 & $\mathrm{NS}$ \\
\hline PCEV & 1445 & 1457,5 & 1335,62 & 1387,5 & 1208,75 & 184,102 & $\mathrm{NS}$ \\
\hline P Cœur & 10,25 & 9,06 & 7,82 & 8,77 & 8,12 & 2,1608 & $\mathrm{NS}$ \\
\hline P foie & $41,87^{\mathrm{a}}$ & $35,39^{\mathrm{ab}}$ & $32,02^{\mathrm{b}}$ & $33,13^{\mathrm{b}}$ & $32,79^{\mathrm{b}}$ & 6,53606 & $*$ \\
\hline PTD & 148,91 & 143,84 & 131,59 & 142,57 & 131,59 & 19,9162 & $\mathrm{NS}$ \\
\hline PGA & 45,24 & 39,19 & 29,83 & 36,48 & 31,28 & 14,1474 & $\mathrm{NS}$ \\
\hline $\begin{array}{c}\text { L } \\
\text { Carcasse }\end{array}$ & 24,93 & 23,5 & 24,06 & 24,75 & 24,12 & 2,5441 & $\mathrm{NS}$ \\
\hline L Cuisse & 21,06 & 20,93 & 19,93 & 19,06 & 19,06 & 1,7682 & $\mathrm{NS}$ \\
\hline T Cuisse & $14,12^{\mathrm{ab}}$ & $15,31^{\mathrm{a}}$ & $14,87^{\mathrm{a}}$ & $14,50^{\mathrm{ab}}$ & $13,56^{\mathrm{b}}$ & 0,95548 & $*$ \\
\hline
\end{tabular}

Les valeurs indicées de lettres distinctes sont significativement différentes $(P<0,05)$ au test de Newman et Keuls.

Poids en gramme $(\mathrm{g})$; Mensuration en centimètre $(\mathrm{cm})$.

Légende : PV : Poids vif ; PCP : Poids de carcasse pleine ; PCEV : Poids de carcasse évidée ; P Cœur : Poids du cœur ; P foie : Poids du foie ; PTD : Poids du tractus digestif ; PGA : Poids du gras abdominal ; L Carcasse : Longueur de la carcasse ; L Cuisse :

Longueur de la cuisse ; $T$ Cuisse : Tour de la cuisse

\section{DISCUSSION}

Gain de poids cumulé, vitesse de croissance et indice de consommation: L'analyse de la variance sur la baisse de GPC révèle des différences significatives à la $2^{\text {ème }}, 3^{\text {ème }}, 6^{\text {ème }}$ et $7^{\text {ème }}$ semaine $(P<0,05)$ et des différences hautement significatives $(P<0,01)$ à la 4 ème et $5^{\text {ème }}$ semaine. Cet effet dépressif pourrait être dû à une utilisation digestive effectuée par les facteurs antinutritionnels contenus dans la féverole. Certains auteurs accusent les tanins qui se combinent avec les protéines et réduisent la rétention de la fraction azotée de la ration et par la suite une réduction de la vitesse de croissance et de l'efficacité alimentaire (Lacassagne et al., 1988 ; Benabdeljejil, 1990 ; Larbier et Leclercq, 1992 ; Kaysi et Melicion, 1992; Metayer et al., 2003). Les facteurs antitrypsiques de la féverole ralentissent la croissance de l'animal (Larbier et Leclercq, 2003). Les PHA ou lectines contenus dans la féverole provoquent des effets défavorables sur le métabolisme et l'utilisation nutritionnelle de cette légumineuse en alimentation et sont souvent auteurs des retards de croissance (Larralde et Martinez, 1991). L'indice de consommation élevé proportionnellement au taux d'incorporation de la féverole peut être dû à l'hypertrophie pancréatique et la réduction de l'efficacité alimentaire causées par les facteurs antitrypsiques de la féverole (Larbier et Leclercq, 1992).
Paramètres pondéraux et conformation de la carcasse : À l'opposé des résultats obtenus par certains auteurs (Guillaume, 1974 ; Larbier et Leclercq, 1992) dans lesquels l'utilisation de la féverole donnait lieu à de meilleurs résultats en phase de finition et les animaux âgés seraient peu sensibles aux effets négatifs des facteurs antinutritionnels éventuellement contenus dans la féverole, les nôtres montrent que le taux d'incorporation de la féverole a un effet dépressif sur poids vifs au taux de $12 \%$. Nos résultats corroborent avec ceux de Metayer et al., 2003 qui indique que l'introduction de la féverole dans la formulation alimentaire inférieure à $12 \%$ en substitution partielle du tourteau de soja n'affecte pas les performances de croissance. Nos travaux n'indiquent aucune différence sur le poids de carcasse pleine, le poids de carcasse évidée et le poids de cœur. La diminution du poids de foie et du pancréas occasionnée par la féverole est également partagée par certains auteurs (Larbier et Leclercq, 2003). Selon eux, cet effet est dû certainement à la réduction de l'efficacité alimentaire. La diminution du poids du gras abdominal s'expliquerait aussi par la diminution du poids du foie, qui réduit le taux de stockage du glycogène d'où mobilisation de la graisse abdominale comme source d'énergie. Cette thèse est confirmée par Lacassagne et 


\section{Okandza et al., J. Appl. Biosci. 2017 Influence de la substitution graduelle de tourteau de soja par la}

féverole sur la croissance et la conformation de la carcasse chez les poulets de chair.

al., 1988 ; et par contre d'autres restent liés à l'influence

\section{CONCLUSION}

À l'issue de ce travail, il est admis que la féverole peut bien être utilisée dans l'alimentation des poulets de chair à des taux bien déterminés. Le taux d'incorporation de la féverole à un effet dépressif sur le poids vif, le poids de carcasse, le poids des organes internes et enfin sur la longueur et le tour de la cuisse. Ces effets sont plus

\section{REFERENCES BIBLIOGRAPHIQUES}

Akouango P, 2014. Évolution qualitative et quantitative des composants de l'œuf pendant les trois phases de ponte chez la poule. Journal of Applied Biosciences 74 :6080-6085

Benabdeljelil K, Boubakraoui, 1985. L'utilisation de la féverole dans l'alimentation du poulet de chair Homme Terre et Eau. Revue Marocaine des Sciences Agronomiques et Vétérinaires. Vol.15(58), 39(52).

Benabdeljalil K, 1990. Des légumineuses en tant que source protéique alternative dans les rations de poulet chair. Institut Agronomique et Vétérinaire Hassan II, Rabat (Maroc). CIHEAM Options Méditerranéennes, Sér. A / nº, 1990 L'aviculture en Méditerranée

Bougon M, 1974. Influence de la substitution du tourteau de Soja par de la féverole sur les performances des pondeuses. Bull St. Exp. Aviculture Ploufragran14 p.102-106

Cartoux P., 2010. Alimentation des bovins viande : Distribuer des pois ou des féveroles. Available from INTERNET : http://www.orne-agri.com

Dragoul C., Raymond G., Marie-Madeleine J., Roland J., Marie-Jacqueline L., Brigitte M., Louis $\mathrm{M}$ et André T -2004. Nutrition et alimentation des animaux d'élevage Tome 1. Page 26-49. Educagri Edition, Dijon. ISBN 978-2-84444-3465.

Fernandez. V, Ruiz. M, 2003. Técnico en ganadería, Volume 1. Técnico en ganadería, Volume 1. Editeur : Culural, 2003. 556 pages.

Franck Y, 1980. In : L'alimentation rationnelle des poulets de chair et des pondeuses. Edition ITAVI.

Guillaume J, 1974. Utilisation de la féverole dans l'alimentation du poulet et de la poule pondeuse $15^{\mathrm{èm}}$ World poultry congress $p .66$

Kaysi $Y$ et Melcion J.P, 1992. Traitements technologiques des protéagineux pour le monogastrique : exemples d'application `à la de la chaleur (Bougon, 1974 ; Larbier et Leclercq, 1992)

marqués à un taux de $12 \%$ de féverole. II est donc nécessaire d'utiliser la féverole dans l'alimentation des poulets de chair sans atteindre $12 \%$ pour ne pas enregistrer des baisses de productivité chez les poulets de chair.

graine de la féverole. INRA Productions animales, 5 (1), pp.3-17.

Lacassagne L., Francesch. M., Carre B And Melcion. J. $P, 1988$. Utilization of tannin containing and tannin-free faba beans (Vicia faba) by young chicks : effects of pelleting feeds on energy, protein and starch digestibility. Anim. Feed Sci. Technol. 20: 59-68.

Larralde J and Et Martinez. J.-A, 1991. Nutritional value of faba bean: effects on nutrient utilization, protein turnover and immunity. Options Méditerranéennes - Série Séminaires 10, 111117

Larbier M et Leclercq B, 1992. Nutrition et alimentation des volailles. Matières premières utilisées en aviculture. Page 255-302. INRA édition.

Larbier M et Leclercq.B. 2003. Nutrition et alimentation des volailles. INRA éditions, Paris : « $p: 63$ 192 »

Lazaro R., García M., Araníbar M. J., Mateos G G, 2003. Effect of enzyme addition to wheat- barley- and rye-based diets on nutrient digestibility and performance of laying hens. Br Poult Sci. 2003 May ; 44(2) :256-65.

Metayer. J, Barrier-Guillot. B, Skiba. F, Crepon. K, Bouvarel. I, Marget. P, Duc. G, Lessire. M, 2003. Valeur alimentaire et utilisation de différents types de féveroles chez le poulet et le coq adulte. Cinquièmes Journées de la Recherche Avicole, Tours, 26 et 27 mars 2003.

Vadivel, V., Pugalenthi, M., 2010. Studies on the incorporation of velvet bean (Mucuna pruriens var. utilis) as an alternative protein source in poultry feed and its effect on growth performance of broiler chickens. Trop. Anim. Health Prod., 42 (7) : 1367-1376

Zitari S, 2008. Étude des valeurs nutritives de certaines ressources alimentaires locales utilisées dans l'alimentation des animaux. Université de Sousse, Master 2008. 